\title{
Jurisdictional Area Minimizing Welfare Loss Based on Accessibility in Transportation Network*
}

\author{
Koji Okuhara $^{\dagger}$, Noriaki KoIDE ${ }^{\dagger}$, Yu ICHIFuJI ${ }^{\ddagger}$ and Noboru SoneHARA ${ }^{\ddagger}$
}

\begin{abstract}
In this paper we discuss about jurisdictional area of fire department minimizing welfare loss based on accessibility in transportation network. Our proposed method can support decision making of assignment problem in the real world by using program with the Google map API. In numerical example, we show that the accessibility of each fire department is higer than the average value of accessibility for all town in its jurisdictional areas. From the result of proposed optimization problem, some towns are shown to assign them to other jurisdictional area than a current jurisdictional area.
\end{abstract}

\section{Introduction}

Spatial model for urban planning have been developed from view of facility location[1,2]. Location of public facilities is important for welfare and service. Especially location of emergency facilities like fire departments and police boxes sometimes affect life of residents. A fire department is a public organization that provides fire protection for a certain jurisdiction, which typically is a prefecture and city.

A fire department usually contains one or more fire stations within its boundaries. The fire department's jurisdiction is organized by the governmental body that controls the department. The most common type of government control is at the municipality level.

Within the jurisdiction the department would setup its organization. This deals with the placement of fire stations, equipment, and personnel within the area of control. For good service, in this paper we discuss about assignment of jurisdiction area of each fire department in certain governmental body.

One of criteria of good service of public facilities is welfare loss which is similar concept like customer loss based on accessibility [3] in economics. This holds equal service to the entire community and gives the assignment of jurisdiction area of department efficient to keep operations.

\footnotetext{
* Manuscript Received Date: May 30, 2012

$\dagger$ Department of Information and Physical Sciences, Graduate School of Information Science and Technology, Osaka University; 1-5 Yamadaoka, Suita, Osaka 565-0871 JAPAN

¥ Information and Society Research Division, National Institute of Information; Hitotsubashi 2-1-2, Chiyoda-ku, Tokyo 101-8430, JAPAN

Key Words: fire department, jurisdiction area, minimizing welfare loss, geographical information system.
}

In this paper, we provide a model which reflects more realistic geographical information by applying estimated distance and time for transportation from GIS. Furthermore we propose new criteria of welfare loss based on accessibility. As the result, our model can include the concept of geographical advantage derived from both distance and time of real world. Such measurement of accessibility has not been considered in usual model.

\section{Facility Location Problem}

\subsection{Geographical Advantage}

Geographical advantage is regarded as the convenience of the location[4,5]. It is important information for considering facility location in the real world. The place where can be accessed easily from other places has strong geographical advantage. When we think the geographical condition about a store and a house we use the word like a geographical convenience. The value of geographical advantage can be given by the index of such geographical convenience, naturally the geographical advantage changes depending on a network structure.

We first consider a graph in which a node represents the cross and an arc represents the road in the proposed network structure. Suppose that there are 7 spots, $\{1,2,3,4,5,6,7\}$, with 12 directed links, $\{1$ $\rightarrow 2,1 \rightarrow 4,1 \rightarrow 5,2 \rightarrow 3,2 \rightarrow 7,3 \rightarrow 4,4 \rightarrow 1,5$ $\rightarrow 1,5 \rightarrow 6,6 \rightarrow 5,7 \rightarrow 1,7 \rightarrow 2\}$.

Let the vector of geographical advantage be

$$
g=\left[\begin{array}{lllllll}
g_{1} & g_{2} & g_{3} & g_{4} & g_{5} & g_{6} & g_{7}
\end{array}\right]^{\mathrm{T}} .
$$

In order to calculate the value of geographical advantage, network structure must be transformed into the adjacency matrix. The element of directed graph satisfies $a_{i j}=1$ if the target node $i$ has an arrow to the other node $j$, otherwise it satisfies $a_{i j}=0$. 


$$
A=\left[\begin{array}{lllllll}
0 & 1 & 0 & 1 & 1 & 0 & 0 \\
0 & 0 & 1 & 0 & 0 & 0 & 1 \\
0 & 0 & 0 & 1 & 0 & 0 & 0 \\
1 & 0 & 0 & 0 & 0 & 0 & 0 \\
1 & 0 & 0 & 0 & 0 & 1 & 0 \\
0 & 0 & 0 & 0 & 1 & 0 & 0 \\
1 & 1 & 0 & 0 & 0 & 0 & 0
\end{array}\right]
$$

The conditions of the value $g_{i}$, which have been listed above, can be represented by using the adjacency matrix as follows:

$$
g_{i}=\sum_{j} \frac{a_{j i}}{\lambda \sum_{k} a_{k i}} g_{j}
$$

The value $g_{i}$ of geographical advantage of node $i$ is thought to satisfy the following conditions.

(1) The value of geographical advantage depends on the shape of road structure, because it is defined by $a_{i j}$.

(2) The geographical advantage is proportional to the total sum of one of neighbor nodes, because $g_{i}$ is derived by total sum of the proportion of $g_{j}$.

(3) It becomes large if the number of connected node increases, because $a_{j i}$ takes 1 if the node $i$ have connection from node $j$.

Then transpose operation is applied to matrix $A$ and get a sum for each row. We assume the transition probability from node $i$ is the same for all connected nodes. Thus modified matrix can be defined as follows:

$$
M=\left[\begin{array}{ccccccc}
0 & 0 & 0 & 1 & 1 / 2 & 0 & 1 / 2 \\
1 / 3 & 0 & 0 & 0 & 0 & 0 & 1 / 2 \\
0 & 1 / 2 & 0 & 0 & 0 & 0 & 0 \\
1 / 3 & 0 & 1 & 0 & 0 & 1 & 0 \\
1 / 3 & 0 & 0 & 0 & 0 & 0 & 0 \\
0 & 0 & 0 & 0 & 1 / 2 & 0 & 0 \\
0 & 1 / 2 & 0 & 0 & 0 & 0 & 0
\end{array}\right],
$$

where the element of matrix $M$ is given by

$$
m_{i j}=\frac{a_{j i}}{\sum_{k} a_{i k}} .
$$

The transition from node $i$ to node $j$ occur based on probability, thus our model can be considered as one of stochastic system. by

Again we can rewrite the condition of the value $g_{i}$

$$
M g=\lambda g .
$$

Now that, we can understand that the derivation of geographical advantage $g$ is the same of finding eigen vector for matrix $M$. The sum of row in matrix $M$ becomes 1, based on Perron-Frobenius theorem, therefore we can find maximum eigenvalue as $\lambda_{\max }=$ 1. The eigen vector for the maximum eigenvalue can be obtained by using power method. The result is as follows:

$$
g=\left[\begin{array}{lllllll}
4.50 & 2.00 & 1.00 & 3.25 & 1.50 & 0.75 & 1.00
\end{array}\right]^{\mathrm{T}} .
$$

By normalizing the eigen vector, a probability vector can be derived by

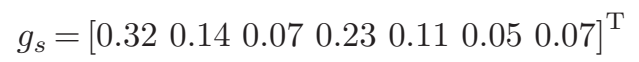

From the results of geographical advantage, we can easily understand node 1 is the most attractive spot from other nodes based on network structure.

\subsection{Concept of Welfare Loss}

In spacial interaction model for facility location, it is clear that to minimize of transportation cost is not only benefit indicator. As one of other indicator of customer's benefit, it may be obtained by integration of demand curve when price becomes change. For example, even if the necessary transportation cost to go to target facility decreases from $p^{(1)}$ to $p^{(2)}$, it can not be assumed that the benefit of customer is the difference $p^{(1)}-p^{(2)}$. Because of a discount of transportation cost, the residents can move easily and it generates new demand to go to the facility.

Mutual effect $r_{o_{i} d_{j}}$ from origin $i$ to destination $j$ in the spacial interaction model given by Table 1 can be considered as one of financial wealth and it supplies benefit to customer side. Good accessibility gives benefit to both customer side and facility side. Based on definition of mutual effect, a customer demand becomes more increase, financial wealth also becomes larger. We call the benefit index of customer which calculated by integration of demand curve the customer's surplus.

Table 1 Information of origin - destination matrix

\begin{tabular}{|c|c|c|c|c|c|c|}
\hline \multirow{2}{*}{$\begin{array}{c}\text { origin } \\
\text { node }\end{array}$} & \multicolumn{5}{|c|}{ destination node } & row \\
\cline { 2 - 6 } & $d_{1}$ & $d_{2}$ & $d_{3}$ & $\cdots$ & $d_{n}$ & sum \\
\hline$o_{1}$ & $r_{o_{1} d_{1}}$ & $r_{o_{1} d_{2}}$ & $r_{o_{1} d_{3}}$ & $\cdots$ & $r_{o_{1} d_{n}}$ & $u_{o_{1}}$ \\
\hline$o_{2}$ & $r_{o_{2} d_{1}}$ & $r_{o_{2} d_{2}}$ & $r_{o_{2} d_{3}}$ & $\cdots$ & $r_{o_{2} d_{n}}$ & $u_{o_{2}}$ \\
\hline$o_{3}$ & $r_{o_{3} d_{1}}$ & $r_{o_{3} d_{2}}$ & $r_{o_{3} d_{3}}$ & $\cdots$ & $r_{o_{3} d_{n}}$ & $u_{o_{3}}$ \\
\hline$\vdots$ & $\vdots$ & $\vdots$ & $\vdots$ & $\vdots$ & $\vdots$ & $\vdots$ \\
\hline$o_{m}$ & $r_{o_{m} d_{1}}$ & $r_{o_{m} d_{2}}$ & $r_{o_{m} d_{3}}$ & $\cdots$ & $r_{o_{m} d_{n}}$ & $u_{o_{m}}$ \\
\hline sum & $v_{d_{1}}$ & $v_{d_{2}}$ & $v_{d_{3}}$ & $\cdots$ & $v_{d_{n}}$ & $T$ \\
\hline
\end{tabular}

Customer's surplus $S$ is assumed to be defined by using a line integration of the demand function $D(p)$ of cost $p$

$$
S=-\int_{p^{(1)}}^{p^{(2)}} D(p) d p .
$$

It can be extended to case that several wealth exist, we have

$$
S=-\sum_{k=1}^{N} \int_{p_{k}^{(1)}}^{p_{k}^{(2)}} D_{k}\left(p_{1}, p_{2}, \cdots, p_{k}, \cdots, p_{N}\right) d p_{k}
$$


where $N$ denotes number of kind of wealth, and suppose that the price of $k$ th wealth changes from $p_{k}^{(1)}$ to $p_{k}^{(2)}[6]$.

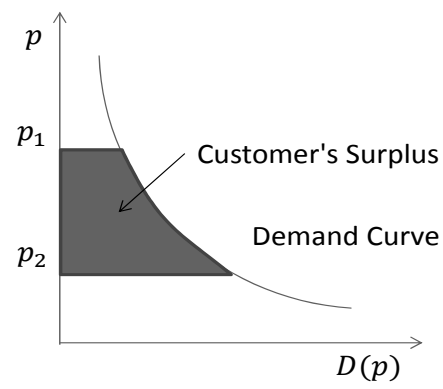

Fig. 1 Concept of customer's surplus in economics

Such a customer's surplus defined as a benefit indicator has a consistency with a discrete choice of resident's behavior from view point of microeconomics.

In this study, the target facilities are one of public sector, therefore if they generate surplus then the customer side will receive the welfare loss. So we focus on the minmization of welfare loss of customer side on formulating model of assignment of jurisdiction area.

Especially in the spacial interaction model, the function satisfies the existence of potential function, thus we can obtain its solution uniquely[7].

In usual spatial interaction model, mutual effect function $r_{o_{i} d_{j}}\left(c_{o_{i} d_{j}}\right)$ of cost $c_{o_{i} d_{j}}$ from origin $i$ to destination $j$ can be derived by maximization of entropy, and it is given by

$$
r_{o_{i} d_{j}}\left(c_{o_{i} d_{j}}\right)=u_{o_{i}} v_{d_{j}} \exp \left(-\beta c_{o_{i} d_{j}}\right)
$$

where $u_{o_{i}}$ and $v_{d_{j}}$ are a row and a column sum of mutual effect functions, respectively[7]. If the distribution of transportation cost is changed from $c_{o_{i} d_{j}}^{(1)}$ to $c_{o_{i} d_{j}}^{(2)}$, then we can define welfare loss of customer as

$$
S_{i j}=\int_{c_{o_{i} d_{j}}^{(1)}}^{c_{o_{i} d_{j}}^{(2)}} r_{o_{i} d_{j}}\left(c_{o_{i} d_{j}}\right) d c_{o_{i} d_{j}}
$$

Here we introduce the interaction for each level $k$ as

$$
r_{o_{i} d_{j}}\left(c_{o_{i} d_{j}}^{(k)}\right)=u_{o_{i}} v_{d_{j}} \exp \left(-\beta c_{o_{i} d_{j}}^{(k)}\right)
$$

The welfare loss of customer can be rewritten by

$$
S=\frac{1}{\beta} \sum_{i=1}^{m} \sum_{j=1}^{n}\left\{r_{o_{i} d_{j}}\left(c_{o_{i} d_{j}}^{(2)}\right)-r_{o_{i} d_{j}}\left(c_{o_{i} d_{j}}^{(1)}\right)\right\}
$$

This means that the welfare loss of customer $S$ is proportional to the difference between levels of mutual effects.

In addition to those characteristics, the following limitation explaining that actual demand can not exceed its potential demand is required.

$$
\frac{\sum_{j=1}^{n} r_{o_{i} d_{j}}\left(c_{o_{i} d_{j}}\right)}{u_{o_{i}}} \leq 1
$$

Furthermore we introduce the $r_{\min }$ as a minimum requirement of acceptable capacity of the facility from view of public section, then the minimumization problem of customer's loss by the spatial interaction model is formulated as follows[8] ;

[Model S]

$$
\begin{array}{ll}
\text { min. } & \sum_{i=1}^{m} \sum_{j=1}^{n} u_{o_{i}} v_{d_{j}} \exp \left(-\beta c_{o_{i} d_{j}}\right) \\
\text { s.t. } & \sum_{j=1}^{n} v_{d_{j}} \exp \left(-\beta c_{o_{i} d_{j}}\right) \leq 1 \\
& \sum_{j=1}^{n} v_{d_{j}} \exp \left(-\beta c_{o_{i} d_{j}}\right) \geq r_{\text {min }} \\
& 0<r_{\text {min }}<1 \\
& v_{d_{j}} \geq 0
\end{array}
$$

\section{Assignment of Jurisdiction Area}

The geographical advantages are added into the minimization problem of welfare loss of customers in spacial interaction model. The objective function of Model S can be rewritten by using the mutual effect $r_{o_{i} d_{j}}\left(c_{o_{i} d_{j}}\right)$

$$
\sum_{i=1}^{m} \sum_{j=1}^{n} u_{o_{i}} v_{d_{j}} \exp \left(-\beta c_{o_{i} d_{j}}\right)=\sum_{i=1}^{m} \sum_{j=1}^{n} r_{o_{i} d_{j}}\left(c_{o_{i} d_{j}}\right)
$$

We derive the first conditional equation as

$$
\frac{\sum_{j=1}^{n} v_{d_{j}} \exp \left(-\beta c_{o_{i} d_{j}}\right)}{v_{d_{k}} \exp \left(-\beta c_{o_{i} d_{k}}\right)} \leq \frac{1}{v_{d_{k}} \exp \left(-\beta c_{o_{i} d_{k}}\right)}
$$

then we take an inverse number from both sides of the equation, and it becomes

$$
\frac{v_{d_{k}} \exp \left(-\beta c_{o_{i} d_{k}}\right)}{\sum_{j=1}^{n} v_{d_{j}} \exp \left(-\beta c_{o_{i} d_{j}}\right)} \geq v_{d_{k}} \exp \left(-\beta c_{o_{i} d_{k}}\right)
$$

The fraction of the left side of the above equation means the ratio of accessibility with respect to total sum of accessibility, let it be $m_{o_{i} d_{j}}$

$$
m_{o_{i} d_{j}}=\frac{v_{d_{k}} \exp \left(-\beta c_{o_{i} d_{k}}\right)}{\sum_{j=1}^{n} v_{d_{j}} \exp \left(-\beta c_{o_{i} d_{j}}\right)}
$$

We can easily understand the ratio $m_{o_{i} d_{j}}$ of accessibility is equal the element of matrix $M$.

Multiplying the geographical advantage $g_{d_{j}}$ and summing up with respect to $j$,

$$
\sum_{j=1}^{n} m_{o_{i} d_{j}} g_{d_{j}} \geq \sum_{j=1}^{n} g_{d_{j}} v_{d_{j}} \exp \left(-\beta c_{o_{i} d_{j}}\right)
$$

Applying the condition of geographical advantage

$$
\sum_{j=1}^{n} m_{o_{i} d_{j}} g_{d_{j}}=g_{o_{i}}
$$

then we have 


$$
g_{o_{i}} \geq \sum_{j=1}^{n} g_{d_{j}} v_{d_{j}} \exp \left(-\beta c_{o_{i} d_{j}}\right)
$$

By using interaction $r_{o_{i} d_{j}}\left(c_{o_{i} d_{j}}\right)$,

$$
v_{d_{j}} \exp \left(-\beta c_{o_{i} d_{j}}\right)=\frac{r_{o_{i} d_{j}}\left(c_{o_{i} d_{j}}\right)}{u_{o_{j}}}
$$

leads the first conditional equation of optimization problem, Model S, becomes

$$
g_{o_{i}} \geq \sum_{j=1}^{n} \frac{r_{o_{i} d_{j}}\left(c_{o_{i} d_{j}}\right)}{u_{o_{j}}} g_{d_{j}}
$$

and the second constraint becomes

$$
g_{o_{i}} \leq \frac{1}{r_{\min }} \sum_{j=1}^{n} \frac{r_{o_{i} d_{j}}\left(c_{o_{i} d_{j}}\right)}{u_{o_{j}}} g_{d_{j}}
$$

Finally we can derive the following optimization problem, Model G, by introducing the geographical advantage into the minimization problem of customer's welfare loss in the spacial interaction model.

$$
\begin{array}{ll}
\text { [Model G] } & \\
\min . & \sum_{i=1}^{m} \sum_{j=1}^{n} r_{o_{i} d_{j}}\left(c_{o_{i} d_{j}}\right) \\
\text { s.t. } \quad & u_{i} g_{i} \geq \sum_{j}^{m} r_{o_{i} d_{j}}\left(c_{o_{i} d_{j}}\right) g_{j} \\
& u_{i} g_{i} \leq \frac{1}{r_{\min }} \sum_{j}^{m} r_{o_{i} d_{j}}\left(c_{o_{i} d_{j}}\right) g_{j} \\
& 0<r_{\min }<1
\end{array}
$$

Here the potential demand $u_{o_{i}}$ is proportional to population at each region and its value is given.

In this study we consider the assignment of jurisdiction area of fire department in Suita city. Suita has four fire departments which are located at east, west, south and north areas. Aim of proposed model is to minimize the welfare loss totally, so objective function is defined by total sum of the welfare loss of customer in four areas.

Let a subscript of the demand point be $N$ and subscript of fire department be $l$. The element of matrix $P_{k I}$ takes 1 or 0 depending on whether the demand point $I$ is assigned to $k$ th fire department or not. The optimization problem is formulated as follows;

\section{[Problem IP]}

$$
\begin{aligned}
& \min . \sum_{k}^{l} \sum_{i=1}^{m} \sum_{j=1}^{n} p_{o_{i} d_{j}}^{(k)} r_{o_{i} d_{j}}\left(c_{o_{i} d_{j}}\right) \\
& \text { s.t. } u_{i} g_{i}{ }^{(k)} \geq \sum_{j}^{m} r_{o_{i} d_{j}}\left(c_{o_{i} d_{j}}\right) g_{j}{ }^{(k)}
\end{aligned}
$$

$$
\begin{gathered}
u_{i} g_{i}{ }^{(k)} \leq \frac{1}{r_{\min }} \sum_{j}^{m} r_{o_{i} d_{j}}\left(c_{o_{i} d_{j}}\right) g_{j}{ }^{(k)} \\
\sum_{k}^{l} p_{o_{i} d_{j}}^{(k)}\left(c_{o_{i} d_{j}}\right)=1 \\
0<r_{\min }<1 \\
p_{o_{i} d_{j}}^{(k)}\left(c_{o_{i} d_{j}}\right)= \begin{cases}1 & \left(\text { if } o_{i}, d_{j} \text { are assigned to } k\right) \\
0 & \text { (otherwise) }\end{cases}
\end{gathered}
$$

We summarize up the procedure of proposed algorithm here.

\section{Procedure of proposed algorithm}

S1 Collection of data of traveling time and distance among all place including the fire departments and towns.

S2 Caluculation of geographical advantage based on estimated transition probability matrix whicih derived from discrete choice theory.

S3 Solving a linear integer programming, Problem IP, with two objective function by using obtained geographical advantages.

\section{Numerical Examples and Discus- sion}

Suita city has 4 jurisdictional areas of fire departments, East, West, South and North areas. The Suita city is covered by jurisdictional areas of 4 fire departments like Figure 2.

Figure 3 shows 47 towns in North fire department's jurisdiction area illustrated by Google Map. Those points can be regarded as a representative place, we can measure a general traveling time and a distance from North fire department.

By using Java programming based on Google Map API, we have measured the traveling time and distance among points for calculating geographical advantages. Figure 4 shows an illustration when the system acquires such information automatically.

Figures 5 and 6 shows the information of both travel time and distance for North and South area, respectively, obtained by Google Map API. Each point describes the travel time versus distance between 2 places. The circle represents data which is relating to its fire department, and the cross represents data for others.

The lines is given by using regression analysis like $y=a x$, where $x$ axis represents travel time and $y$ axis represents distance.

We can design the element of matrix $M$ based on such information reflecting the real world. For example, the matrix for North area consists of $47 \times 47$ elements. Of course the diagonal elements take 0 since they mean the travel time of self loop. By calculating geographical advantages for evaluating the accessibility by traveling time, the result are shown in Table 2 . 


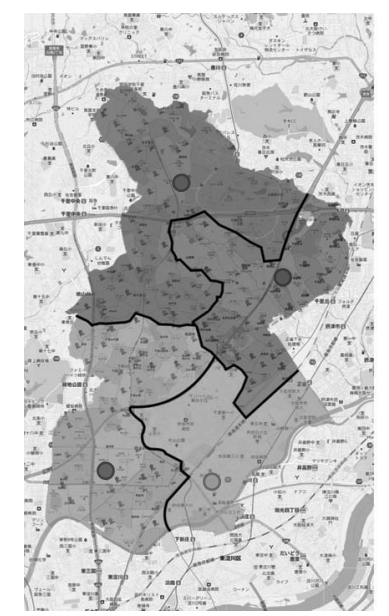

Fig. 24 jurisdictional areas of each fire department of Suita city

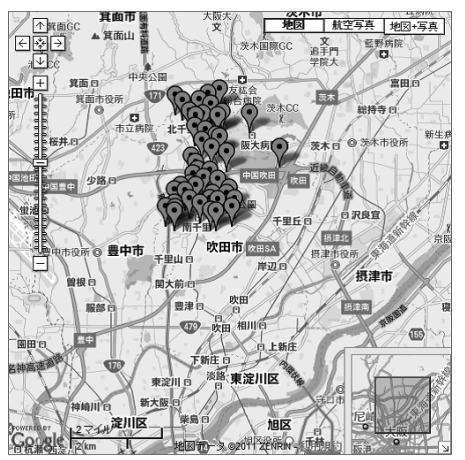

Fig. 347 places in east fire department's jurisdiction area

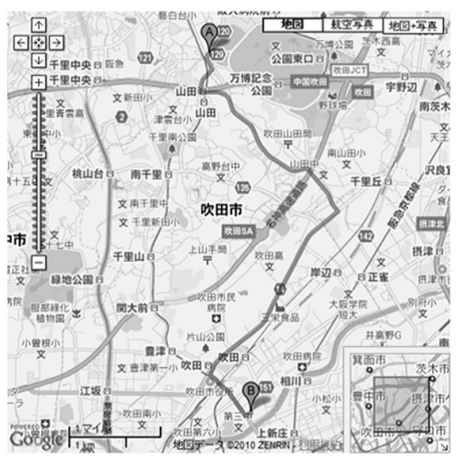

Fig. 4 Route, traveling time and distance in the real world

\section{[Main result I]}

Average value of accessibility for all point in each jurisdictional areas is lower than one of its fire department, thus it can be easily recognized that its geographical advantage of fire department is taking higher value than other place of town.

This means that the east fire department is located in good place to access to other town in current situation. However, the facility location of fire department in South area is better than North area, such trend can be recognized from Figures 5 and 6 .

\section{[Main result II]}

The result of solving optimization problem is shown in Figure 7. Compared with Figure 2, it is found that there are some changes of jurisdictional

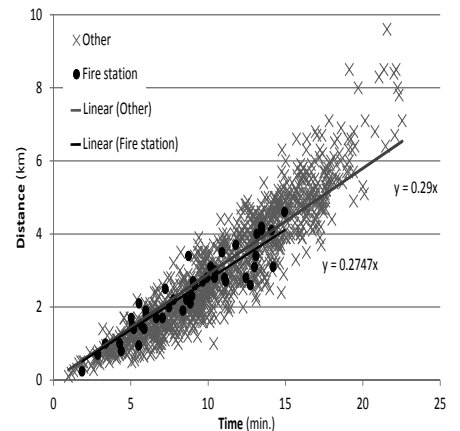

Fig. 5 Distance and travel time between 2 points in North area

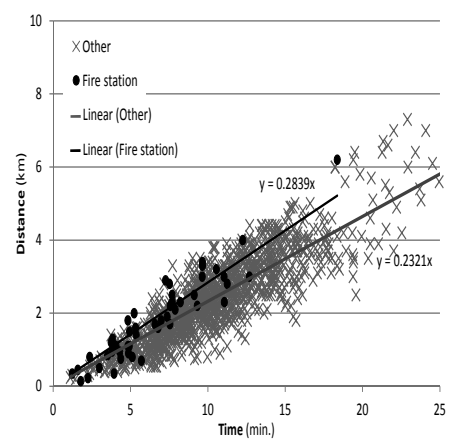

Fig. 6 Distance and travel time between 2 points in South area

Table 2 Accessibility for 4 fire departments

\begin{tabular}{|c|c|c|c|c|}
\hline \multirow{2}{*}{} & \multicolumn{4}{|c|}{ Fire department } \\
\cline { 2 - 5 } & East & West & South & North \\
\hline Accessibility & 0.9652 & 0.955 & 1.000 & 0.919 \\
\hline Ranking & 2 nd & 3rd & Best & Worst \\
\hline
\end{tabular}

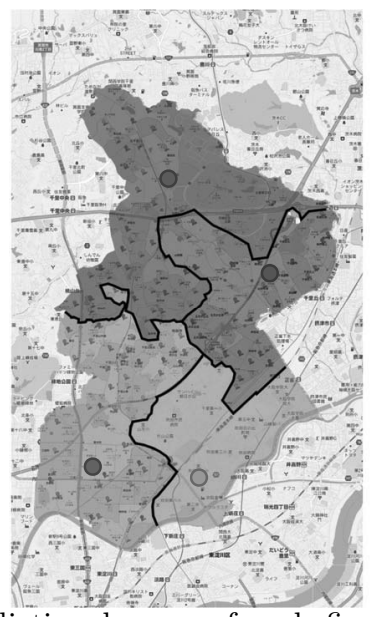

Fig. 74 jurisdictional areas of each fire department of Suita city derived by proposal algorithm

area, Aoba-kita should be assigned from East to North, Saidera-4-chome from West to North, Yamatecho-3chome from South to West and Momoyamadai-1chome from North to West, respectively. One of them becomes an isolated enclave because jurisdiction is determined by accessibility based on road. 


\section{Conclusions}

In this paper we refine usual formulation of optimization problem for facility location of public sector by introducing the concept of geographical advantage. The minimization of welfare loss of customer is one of important criteria of evaluation. By using geographical information system like Google map API, we develop an optimization problem for assignment of jurisdiction area to target facility. Furthermore in numerical example, we show our proposed method can support decision making of assignment problem in the real world.

\section{Acknowledgements}

The authors would like to gratefully acknowledge the helpful discussion of Mr. M. Yamanaka, which have obtained results of numerical examples.

\section{References}

[1] T. Drezner and Z. Drezner: Competitive facilities: market share and location with random utility; Journal of Regional Science, Vol. 36, No. 1, pp. 1-15 (1996)

[2] R. Aboolian, O. Berman and D. Krass: Competitive facility location and design problem; European Journal of Operational Research, Vol. 182, No. 1, pp. 40$62(2007)$

[3] K. Okuhara, K. Y. Yeh, J. Shibata, H. Ishii and H. C. Hsia: Evaluation and assignment of traffic volume for urban planning based on planner and user stance; International Journal of Innovative Computing, Information and Control, Vol. 4, No. 5, pp. 1151-1160 (2008)

[4] H. Noda: The advantage of position as a quantitative criterion according to the characteristics of street network: Quantitative analysis of city planning using the characteristics : of street network Part 1; Journal of Architecture, Planning and Environmental Engineering, Transactions of AIJ, Vol. 470, pp. 139-148 (1995)

[5] T. Ukai and O. Kurita: A theory of geographical significance based on spatial interaction; Journal of Architecture and Planning, Transactions of AIJ, Vol. 616, pp. 129-135 (1995)

[6] H. Tanimura, H. Kaji, S. Ikeda and T. Koshizuka: Urban Planning Mathematics, Asakura (1986)

[7] A. G. Wilson: Entropy in Urban and Regional Modelling, Pion (1970)

[8] H. Tanimura: Duality of the spatial interaction model and its application to the location-allocation problem; Journal of architecture and planning, Transactions of AIJ, Vol. 319, pp. 98-108 (1982)

\section{Authors}

\section{Koji OKuhara (Member)} See p. 67

Noriaki KoIDE

See p. 67

Yu ICHIFUJI

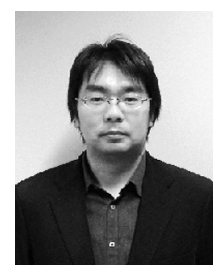

$\mathrm{Yu}$ Ichifuji is a project researcher of Transdisciplinary Research Integration Center, at the National Institute of Informatics since 2010. He received a B.E., M.E., and Ph.D in Information Sciences from Tohoku University, Japan in 2003, 2005 and 2010 respectively. His current research topics are Privacy, Risk management, Resilience, tourism and Web data mining. He is a member of IPSJ and IEICE.

Noboru SoneHARA

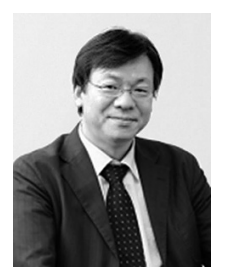

Noboru Sonehara is a Professor of the Information and Society Research Division, at the National Institute of Informatics since 2004. Previously, he was a project manager, Content Commerce Project, at NTT Cyber Solutions Laboratories from 2001 to 2004. He received a BE and ME from Shinshu University, Japan in 1976 and 1978, respectively. He received a $\mathrm{PhD}$ in 1994. He has been a Director of the Information and Society Research Division since 2006. His current research topics are ICT Security, Privacy, Trust, Risk, Resilience, and e-Authentication platform. 